\title{
Resonant Control of a Single-Phase Full-Bridge Unity Power Factor Boost Rectifier
}

\author{
R. Costa-Castelló, R. Griñó, E. Fossas
}

\begin{abstract}
This work studies the dynamics of a single-phase full-bridge unity power factor boost AC-DC converter and develops a linear cascade resonant controller in order to meet two control objectives: to regulate the mean value of its DC output voltage and to drain from the network a sinusoidal current in phase with the sinusoidal source voltage, i.e. keeping the input power factor close to unity. The controller has an inner current control loop with a bank of passive resonators working in parallel that contribute with a high gain to track the desired current reference. The outer control loop takes care of the regulation of the mean value of the DC output voltage of the converter. The feedback channel of this loop includes a filter to attenuate the intrinsic oscillations of the output voltage. Some numerical experiments are included to show the good behavior of the closed-loop system.
\end{abstract}

\section{INTRODUCTION}

In order to meet the requirements of the electrical quality standards (for example IEC 1000-3-2) for low-power equipment, it is necessary to perform the AC-DC conversion of the electrical power using switch-mode power converters [4]. Among these circuits, the most popular choice for medium and high power applications is the boost power converter operating in continuous conduction mode [1], [5]. However, as it is known, it is difficult to control these converters because of their non-minimum phase behavior with respect to the output voltage. This fact is worsened by the basic control objective of the sinusoidal shape in the converter input current. This specification has a non-standard form because it only imposes the shape of a signal and not its value as a function of time, which would fit to a tracking problem. Also, there is another control objective: the mean value of the DC bus capacitor voltage must be regulated to a specified value. But, as it will be seen in the next section, the two control objectives must accomplished with only one control variable, and this fact implies a complex controller structure, in general, a two control loop topology.

The paper is organized as follows: section II presents the converter physical model and the control objectives, section III discusses the steady-state and the zero-dynamics behavior of the system; section IV introduces the resonator concept and its main characteristics; section $\mathrm{V}$ describes the controller architecture and design; section VI shows some numerical experiments. Conclusions are in section VII.

This work was supported in part by the Ministerio de Educación y Ciencia (MEC) under project DPI2004-06871-C02-02.

The authors are with the Instituto de Organización y Control de Sistemas Industriales, Universitat Politècnica de Catalunya, Barcelona, Spain. Email:\{ramon.costa,roberto.grino,enric.fossas\}@upc.edu

\section{Problem formulation}

\section{A. Physical model of the boost converter}

The averaged model of the boost converter (at the switching frequency) [2] is given by

$$
\begin{aligned}
L \dot{x}_{1} & =-u x_{2}-r x_{1}+v_{s} \\
C \dot{x}_{2} & =u x_{1}-\frac{1}{R} x_{2}
\end{aligned}
$$

where $x_{1}$ and $x_{2}$ are the input inductor current and the output capacitor voltage variables, respectively; $v_{s}=E \sin \left(\omega_{r} t\right)$ is the ideal sinusoidal source that represents the AC-line source; $R$ is the DC-side resistive load; $r$ is the parasitic resistance of the inductor; and $L$ and $C$ are the inductance and the capacitor of the converter. The control variable $u$ takes its value in the closed real interval $[-1,1]$ and represents the averaged value of the PWM (pulse-width-modulated) control signal injected into the real system.

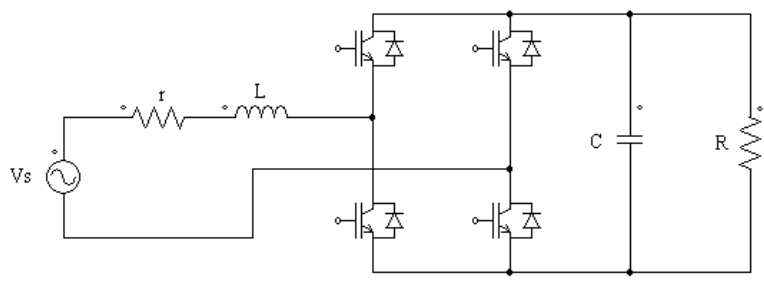

Fig. 1. Bidirectional boost active rectifier converter.

In the actual implementation of the system it is assumed that the output voltage $x_{2}$, the input current $x_{1}$ and the source voltage $v_{s}$ are available for measurement. In the following analysis, it will be interesting to deal with the DC component $^{1}$ of some variables that will be noted as $\langle\cdot\rangle_{0}$. It is important to remark that the system described by equations (1) and (2) can be seen as the interconnection of two subsystems with different time constants. In particular, the dynamics of equation (2) is much slower than the dynamics of equation (1). This fact has led to the development of the classical control schemes for these systems consisting of two concentric control loops: the inner (fast) for shaping the inductor current, and the outer (slow) for regulating the output capacitor voltage. In this control architecture, the output of the outer loop controller acts as the modulating

\footnotetext{
${ }^{1}$ The DC component, or averaged function, of a periodic signal $f(t)$ of period $T$ is calculated as $\langle f(t)\rangle_{0} \triangleq \frac{1}{T} \int_{t-T}^{t} f(\tau) d \tau$.
} 
TuA04.3

signal in an AM modulator, with carrier $v_{s}$, whose output is the reference for the inner loop. The handicap of this control topology, caused by the slow outer voltage loop, is the need for big capacitors in the DC bus to prevent large overvoltages in case of great load perturbations.

\section{B. Control objectives}

The control objectives are:

1) The AC-DC converter must operate with a power factor close to one. This is achieved by ensuring that, in the steady-state, the inductor current $x_{1}$ follows a sinusoidal signal with the same frequency and phase as the AC-line voltage source $v_{s}$, i.e. $x_{1 d}=I_{d} \sin \left(\omega_{r} t\right)$. The value for $I_{d}$ should be calculated by the controller in order to accomplish the following objective,

2) The DC component of the output capacitor voltage $\left\langle x_{2}\right\rangle_{0}$ should be driven to the constant reference value $\left\langle x_{2}\right\rangle_{0 d}$, where $\left\langle x_{2}\right\rangle_{0 d}>E$ in order to have boost behavior [2].

3) The value of the DC bus capacitor must be as low as possible for cost reasons. This requirement implies that the controller should be able to reject large perturbations in the load with short transients to prevent overvoltages in the bus.

\section{Steady-State and Zero-Dynamics Analysis}

As it is known, the bidirectional boost rectifier has relative degree 1 regardless of the output, $x_{1}$ or $x_{2}$. Besides this, it is also known that if the output is $x_{2}$, the system has a non-minimum phase behaviour. For this reason, this system is usually controlled through the current $x_{1}$. In this case, the system has a minimum phase behaviour, i.e. its zerodynamics is stable. In order to verify this assertion, $x_{1}$ is taken as the output of the system by fixing its value to $x_{1 d}=$ $I_{d} \sin \left(\omega_{r} t\right)$ in equations (1)-(2) resulting in

$$
\begin{aligned}
\overline{u x_{2}}= & \left(E-r I_{d}\right) \sin \left(\omega_{r} t\right)-\omega_{r} L I_{d} \cos \left(\omega_{r} t\right) \\
C \frac{d \bar{x}_{2}}{d t}= & \frac{-I_{d}^{2} \omega_{r} L \sin \left(\omega_{r} t\right) \cos \left(\omega_{r} t\right)}{\bar{x}_{2}}-\frac{I_{d}^{2} r\left(\sin \left(\omega_{r} t\right)\right)^{2}}{\bar{x}_{2}} \\
& +\frac{I_{d} E\left(\sin \left(\omega_{r} t\right)\right)^{2}}{\bar{x}_{2}}-\frac{\bar{x}_{2}}{R}
\end{aligned}
$$

where $\bar{u}$ and $\bar{x}_{2}$ are the control variable and the capacitor voltage, respectively, in the zero-dynamics. Then, equation (4) describes the behaviour of the zero-dynamics of the system. This equation is a Bernoulli ODE, but multiplying each side of the equation (4) by $\bar{x}_{2}$ and taking $\bar{z}=\bar{x}_{2}^{2}$, we get the following linear ODE:

$$
\begin{aligned}
\frac{C}{2} \frac{d \bar{z}}{d t}= & -I_{d}^{2} \omega_{r} L \sin \left(\omega_{r} t\right) \cos \left(\omega_{r} t\right)-I_{d}^{2} r\left(\sin \left(\omega_{r} t\right)\right)^{2} \\
& +I_{d} E\left(\sin \left(\omega_{r} t\right)\right)^{2}-\frac{\bar{z}}{R}
\end{aligned}
$$

whose solution is $\bar{z}(t)=f(t)+p(t)+K$, where

$$
f(t)=C_{1} \exp \left(-\frac{2 t}{R C}\right)
$$

TABLE I

CENTER AND RADII OF THE STRIP WHERE $x_{2}$ EVOLVES.

\begin{tabular}{||l|c|r||}
\hline \hline$C$ & $\sqrt{K}$ & $\frac{\sqrt{A^{2}+B^{2}}}{2 \sqrt{K}}$ \\
\hline 0.00099 & 399.99 & 6.45 \\
\hline 0.0099 & 399.99 & 0.64 \\
\hline 0.099 & 399.99 & 0.06 \\
\hline \hline
\end{tabular}

is the vanishing $\left(\lim _{t \rightarrow \infty} f(t)=0\right)$ term corresponding to the first order linear dynamics,

$$
p(t)=A \sin \left(2 \omega_{r} t\right)+B \cos \left(2 \omega_{r} t\right)
$$

is the oscillating term (at frequency $2 \omega_{r}$ ), and

$$
K=\frac{R}{2}\left(E I_{d}-r I_{d}^{2}\right)
$$

is the constant term. Furthermore, $\|\bar{z}\|_{2}^{2}$ is equal to

$\frac{I_{d}^{2} R^{2}}{4}\left(\left(E-r I_{d}\right)^{2}\left(1+\frac{1}{1+C^{2} \omega_{r}^{2} R^{2}}\right)+\frac{\omega_{r}^{2} I_{d}^{2} L^{2}}{1+C^{2} \omega_{r}^{2} R^{2}}\right)$.

As a conclusion, the output voltage $x_{2}$ is periodic and evolves in the strip approximately centered at $\sqrt{K}$ with radius

$$
\frac{\sqrt{K+\sqrt{A^{2}+B^{2}}}-\sqrt{K-\sqrt{A^{2}+B^{2}}}}{2} \simeq \frac{\sqrt{A^{2}+B^{2}}}{2 \sqrt{K}}
$$

where

$$
\sqrt{A^{2}+B^{2}}=\frac{I_{d} R}{2 \sqrt{1+C^{2} \omega_{r}^{2} R^{2}}} \sqrt{\left(E-r I_{d}\right)^{2}+I_{d}^{2} \omega_{r}^{2} L^{2}}
$$

Approximation values for the strip center and radius for several values of capacitor $C$ are given in Table I. The remaining converter parameters are $E=230 \sqrt{2.0} \mathrm{~V}, \omega=$ $100 \pi$ Hertz, $L=0.008 \mathrm{H}, R=100 \Omega, r=0.5 \Omega, V_{d}=400 \mathrm{~V}$.

Steady-state dynamics is $\frac{2 \pi}{\omega_{r}}$-periodic in both variables $x_{1}$ and $x_{2}$, so does the energy $H=L x_{1}^{2}+C x_{2}^{2}$ stored in the converter and its derivative. From equations (1)-(2)

$$
\frac{d H}{d t}=v_{s} x_{1}-r x_{1}^{2}-\frac{x_{2}^{2}}{R}
$$

Thus, integrating the periodic function $\frac{d H}{d t}$ along one period yields to

$$
0=\int_{t-T_{r}}^{t} v_{s}(\tau) x_{1}(\tau)-r x_{1}^{2}(\tau) d \tau-\int_{t-T_{r}}^{t} \frac{x_{2}^{2}(\tau)}{R} d \tau
$$

Hence,

$$
\int_{t-T_{r}}^{t} v_{s}(\tau) x_{1}(\tau)-r x_{1}^{2}(\tau) d \tau-\int_{t-T_{r}}^{t} \frac{x_{2}^{2}(\tau)}{R} d \tau
$$

that can be read as the input active power is equal to the output active power and results in

$$
\frac{R I_{d}}{2}\left(E-r I_{d}\right)=\left\langle x_{2}^{2}\right\rangle_{0} .
$$

For high values of the capacitor $C,\|\bar{z}\|_{2}^{2}$ can be approximated by

$$
\frac{I_{d}^{2} R^{2}}{4}\left(E-r I_{d}\right)^{2}
$$


Hence, taking $C>>0, \bar{z}$ can be reduced to its DC component,

$$
\bar{z} \simeq\langle\bar{z}\rangle_{0} \simeq\left\langle x_{2}\right\rangle_{0}^{2}=V_{d}^{2}
$$

where $V_{d}$ is the output voltage desired value. This provides a nominal value for $I_{d}$. Namely,

$$
\frac{R I_{d}}{2}\left(E-r I_{d}\right)=V_{d}^{2}
$$

This equation has two solutions $I_{d}=\frac{E}{2 r} \pm \sqrt{\frac{E^{2}}{4 r^{2}}-\frac{2 V_{d}^{2}}{r R}}$ which are real if and only if $\frac{V_{d}}{E}<\sqrt{\frac{R}{8 r}}$ [2]. This condition is known as the boost condition of the power converter. The smaller solution of (8),

$$
I_{d}=\frac{E}{2 r}-\sqrt{\frac{E^{2}}{4 r^{2}}-\frac{2 V_{d}^{2}}{r R}},
$$

corresponds to a stable equilibrium and is the selected relation between the desired mean value of the DC capacitor $\left(V_{d}\right)$ and the amplitude of the desired inductor current $\left(x_{1 d}=\right.$ $\left.I_{d} \sin \left(\omega_{r} t\right)\right)$.

Similar results can be obtained averaging equation (5),

$$
\frac{C}{2} \frac{d\langle\bar{z}\rangle_{0}}{d t}=\frac{\left(E I_{d}-r I_{d}^{2}\right)}{2}-\frac{\langle\bar{z}\rangle_{0}}{R}
$$

whose solution is

$$
\langle\bar{z}\rangle_{0}=\frac{R}{2}\left(E I_{d}-r I_{d}^{2}\right)+C_{2} \exp \left(-\frac{2 t}{R C}\right) .
$$

IV. IMP BASED CONTROL

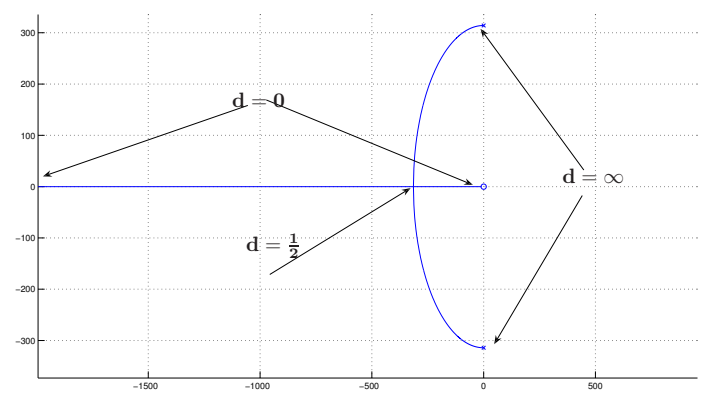

Fig. 2. Zero Root Locus for PR internal models (zeros as function of $d$ ) for $\omega=10$.

According to the Internal Model Principle [3] a necessary and sufficient condition to asymptotically track or reject signals in a closed-loop systems is introducing inside the loop the signal generator. We are interested in tracking and rejecting sinusoidal signals. The linear generator of this type of signals, commonly named resonator, is characterized by having a denominator $s^{2}+\omega_{r}^{2}$ when written in the Laplace transform domain. A set of resonators in parallel, tuned at appropriate frequencies will be considered in this paper for control purposes.

In this section some basic properties of resonators will be stated. We emphasize on frequency ones because of closed loop stability will be justified in the Nyquist domain.

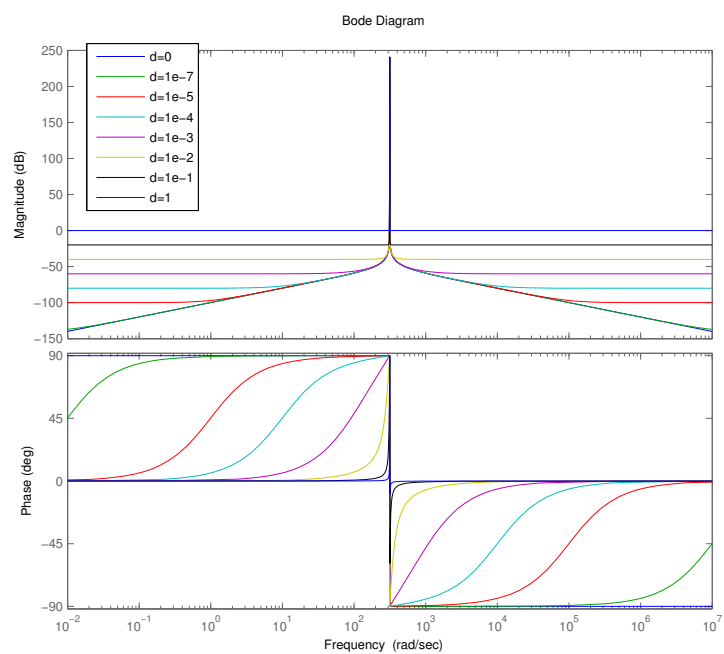

Fig. 3. Resonator gain-phase for $\omega=10$ and different values of $d$.

Transfer functions with with relative degree 1 that are internal models for sinusoidal signals with frequency $\omega_{r}$ can be written as

$$
R(s)=k \frac{s+\alpha}{s^{2}+\omega_{r}^{2}}
$$

In order to determine which of these transfer functions are positive real, let us to compute

$$
\Re\{R(j \omega)\}=k \frac{\alpha}{\omega_{r}^{2}-\omega^{2}}
$$

To guarantee that this function is positive for $\omega \in[0, \infty)$ from 0 it is necessary that $\alpha=0$. Hence, all relative degree 1 positive real internal models have the form

$$
R(s)=k \frac{s}{s^{2}+\omega_{r}^{2}}
$$

Clearly, all functions with an added proportional path

$$
R(s)=k \frac{s}{s^{2}+\omega_{r}^{2}}+k \cdot d=k \frac{d s^{2}+s+d \omega_{r}^{2}}{s^{2}+\omega_{r}^{2}}
$$

with $d>0$ are also positive real. This functions correspond to relative degree 0 internal models. Fig. 2 shows the zero root locus of these transfer functions depending on the value of $d$. Note that for small values of $d$, the zeros are 0 and $\infty$ as it can be seen in equation (11). This zeros keep real until $d=\frac{1}{2 \omega_{r}}$ is reached, at this value a bifurcation exists and the two zeros equal to $-\omega_{r}$. From this point as $d$ increases the zeros asymptotically tend to the poles $\pm w_{r} \cdot j$ following a quadratic trajectory.

Fig. 3 shows the gain-phase plot of the resonators for several values of $d$. Note that DC gain is $k \cdot d$, this is also the gain at $\omega=\infty$. As it can be seen in Fig. 3 for values of $d$ greater than 1 the gain stays close to $k \cdot d$ in all frequency range except close to $w_{r} \mathrm{rad} / \mathrm{s}$ where very high gains are obtained.

For control purposes, a set of resonators at frequencies multiple of $\omega_{r}$ will be added in parallel, in the closed loop. Note that this parallel set of positive real transfer functions are positive real as well. 


\section{Control Design}

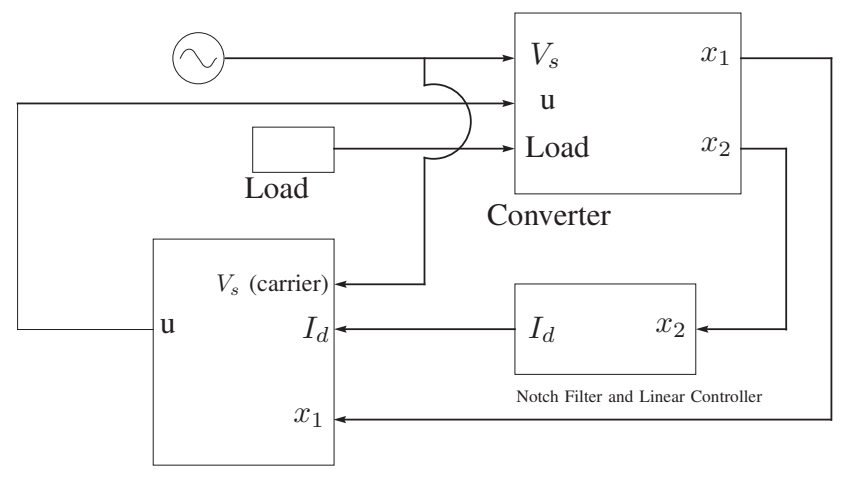

Resonant Controller

Fig. 4. Control scheme block diagram.

The control objectives are

- $x_{1}(t)=I_{d} \sin \left(\omega_{r} t\right)$, and

- $\left\langle x_{2}\right\rangle_{0}=V_{d}$

in steady-state. In order to meet these goals a cascade controller is used. The inner control loop is in charge of shaping the network current and the outer control loop is responsible for assuring the desired DC bus voltage mean value. Fig. 4 shows the block diagram of the complete control system that is detailed in the following subsections.

\section{A. Current Controller}

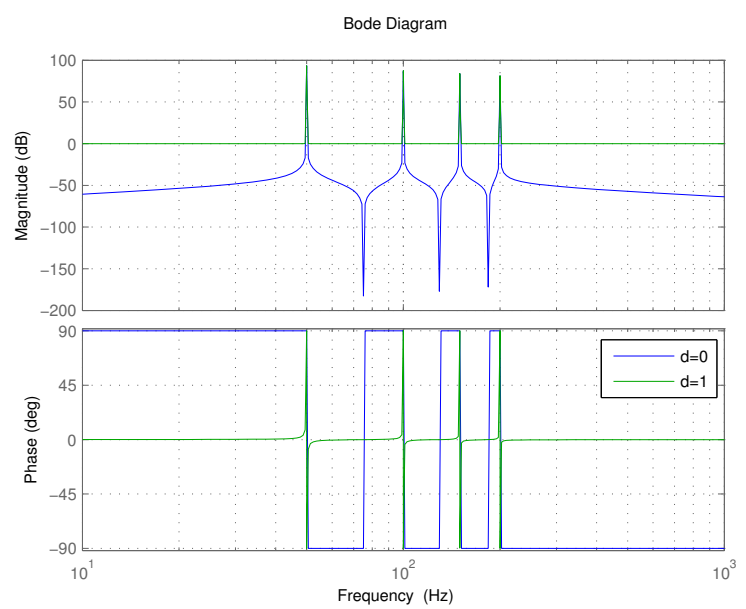

Fig. 5. Resonator bank frequency response with four resonators with $\omega_{k}=2 \pi 50 k \mathrm{rad} / \mathrm{s}, k=1,2,3,4$ and $d \in\{0,1\}$.

In this section, a controller which forces the network current to have the desired sinusoidal signal shape will be designed. Although equation (2) is nonlinear, a common practice is to use a linearized version (around the desired constant average value of the DC bus voltage $\mathrm{Vd}$ ) to design the controller. The transfer function that describes its dynamic behavior is

$$
G_{p}(s)=\frac{x_{1}(s)}{u(s)}=\frac{-V_{d}}{L \cdot s+r}
$$

that corresponds to a first order linear system. Clearly, its phase plot in the Bode diagram, apart from the minus sign ${ }^{2}$, lies in the interval $\left[-\frac{\pi}{2}, 0\right] \mathrm{rad}$. Thus the system is positive real.

In the actual system, $x_{2}$, in steady-state, is a periodic signal of period $\frac{2 \pi}{\omega_{r}}$ being $\omega_{r}$ the network frequency $(50 \mathrm{~Hz}$ in this work). So, the harmonics introduced through $x_{2}$ are situated at frequencies that are integer multiples of $\omega_{r}$. Hence, a parallel set of resonators $R_{k}(s)$ (a resonator bank) at frequencies $k \omega_{r}, k=1, \ldots, n$ will be used as a controller. The term $d_{k}$ is necessary to assure closed-loop stability. All these proportional terms can be grouped in a single one and the full resonator bank can be written as

$$
R(s)=d+\sum_{k} R_{k}(s)=d+\sum_{k} \frac{s}{s^{2}+(k \omega)^{2}}
$$

An example of the obtained frequency response for $\omega_{r}=$ $2 \pi 50 \mathrm{rad} / \mathrm{s}, d=1$, using 4 resonators $\left(\omega_{k}=k \omega_{r}, k=\right.$ $1,2,3,4)$ is shown in Fig. 5. The frequency response of $R(s)$ can be seen as a constant gain (equal to $d$ ) with infinite gain at the frequencies where the resonators have been placed. Something similar happens with the phase, which keeps close to 0 in all the frequencies except in those where the resonators have been placed. This simplified frequency response can be of great interest to analyze and design the closed-loop system. In this approach $d$ remains as the only design parameter once the desired harmonic frequencies have been fixed.

The frequency and the number of resonators depend on the specific applications. The later number is related with the harmonics which may appear on the network current. For simplicity only 3 resonator will be used in this work.

\section{B. Voltage Controller}

This section deals with the design of the controller (outer control loop) to meet the second control objective, i.e. the DC bus voltage mean value $\left\langle x_{2}\right\rangle_{0}$ must be regulated to $V_{d}$, applying classical linear control design to the linearized model of equation (2) where $x_{1}=I_{d} \sin \left(\omega_{r} t\right)$ and $I_{d}$ acts as a control variable.

Equation (9) offers a straightforward solution. But its use as the only control action has no practical sense due to the parametric uncertainty of the plant. Additionally, equation (9) requires the knowledge of $R$ which is usually unknown or time varying due to possible load changes in real operation. A solution to this last uncertainty problem might be the introduction of an estimator for $R$ but, in this work, a feedforward and a feedback combined actions is chosen as alternative approach for the control. In this manner, the whole controller would be robust against the plant and load uncertainty.

\footnotetext{
${ }^{2}$ This minus sign will be compensated by a corresponding one in the controller.
} 
The feedforward action corresponds to (9) evaluated for the nominal value of the load, $R$, and the rest of the plant parameters. The feedback action that is in charge of compensating the uncertainties in the plant and the load changes consists of a negative feedback loop with a classical PI controller with an anti-windup mechanism to prevent saturation problems when the current amplitude $I_{d}$ exceeds the maximum allowed value. This controller will regulate $\left\langle x_{2}\right\rangle_{0}$ to the desired value $V_{d}$ without steady-state error and it will attenuate step changes in the load. Besides this, it also compensates the parasitic losses in the inductor and capacitor parasitic resistances ${ }^{3}$.

It is important to remark that in steady state, $x_{2}$ has a DC component (useful component) and an harmonic content at frequencies $k \omega_{r}, k>1$. The harmonic content is intrinsic to the operation of the converter with near unity power factor and it can not be reduced with any control action, in fact, to reduce the amplitude of this harmonic content the DC bus capacitor value must be increased. However, this harmonic content will affect negatively to the performance of the controlled system since the voltage oscillations will propagate through the voltage controller to the reference current. In order to overcome this problem a possible solution is to reduce the bandwidth of the voltage controller but this option would reduce the good performance of the system in front of load changes. So, in this work, it was chosen to pass the $x_{2}$ feedback signal through a conditioning stage that consists of a comb filter to reject this unwanted harmonic content and leave the DC component unchanged. In particular, the transfer function of the comb filter is

$$
H(s)=\frac{1}{T} \frac{1-e^{-s T}}{s}
$$

where $T=\frac{2 \pi}{\omega_{r}}$ is the fundamental period of the signal $x_{2}$. Then, the use of this filter improves the transient response in front of load changes because it allows to increase the bandwidth of the voltage controller.

\section{Numerical Simulations}

To validate the theoretical developments a number of numerical simulations have been performed. In this simulations the following parameters have been used: $L=0.8 \mathrm{mH}$, $C=9.9 \mathrm{mF}, r=0.5 \Omega$ and $R=100 \Omega$. The value of $C$ and $L$ has been designed in order to obtain steady-state oscillations which are less than $1 \%$ in the DC bus when regulated to $400 \mathrm{~V}$ and using the specificed $R_{L}$. This design is based on the bounds obtained in equation (7).

The experiments which have been performed consist of starting the system without load, plugging the load, $R$, at time $t=5 \mathrm{~s}$, plugging a second $R$ at time $t=10 \mathrm{~s}$, and finally unplugging one of this resistive loads at $t=15 \mathrm{~s}$. Obtained results are shown in Figs. 6, 7, 8, 9, 10 and 11. Fig. 6 corresponds to output of the voltage controller, note that there is a very short transient for $I_{d}$, which value in

\footnotetext{
${ }^{3}$ These uncertainties can be also considered as additive complex-dynamics disturbances that will be compensated in the frequency range of the openloop bandwidth of the voltage control system.
}

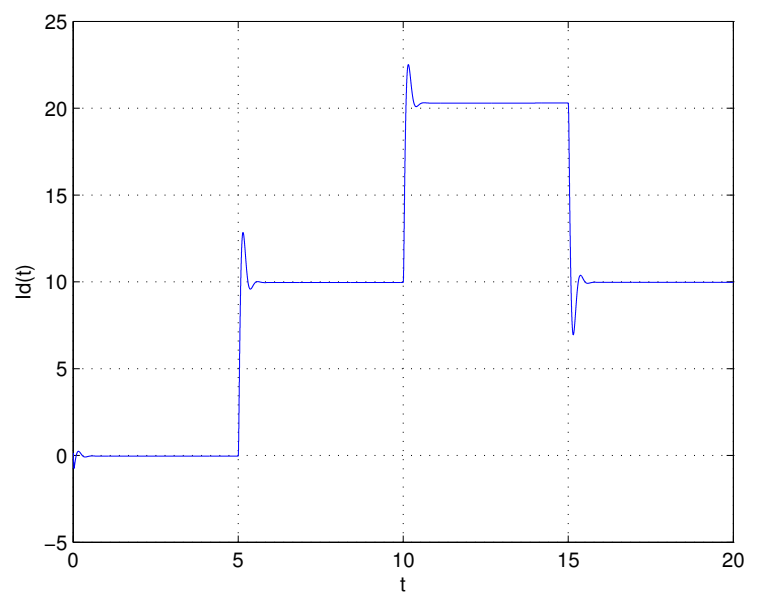

Fig. 6. Evolution of $I_{d}$ during the simulation.

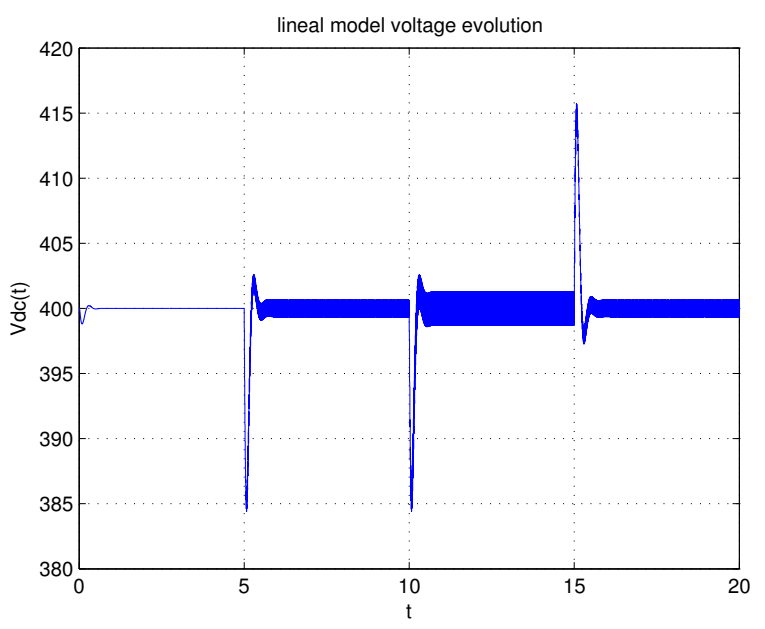

Fig. 7. Evolution of $V_{D C}$ during the simulation.

steady-state almost equal the one predicted in 9. Fig. 7 shows the evolution of the DC bus voltage. After the system initialization the mean value remains constant around the desired mean value. This figure clearly shows the nonminimum phase characteristic of the system. As previously stated, the DC bus in steady state is composed by a DC component and a harmonic one, this harmonic is shown in detail in Fig. 8, it can be seen that the oscillations frequency is $100 \mathrm{~Hz}$, twice the voltage source one. The amplitude of this oscillation has been designed when selecting $C$ and $L$ using equation (7) so that it lies below the 1\%. Fig. 9 shows the evolution of the net current during the complete experiment. Note that the amplitude of this current is the one consigned by $I_{d}$ and looking at Fig. 10 it can be seen that it perfectly tracks the desired reference in steady state. Finally Fig. 11 shows the control action evolution, it is important to note that this variable is always in the $[-1,1]$ interval so proposed controlled is always running properly. 


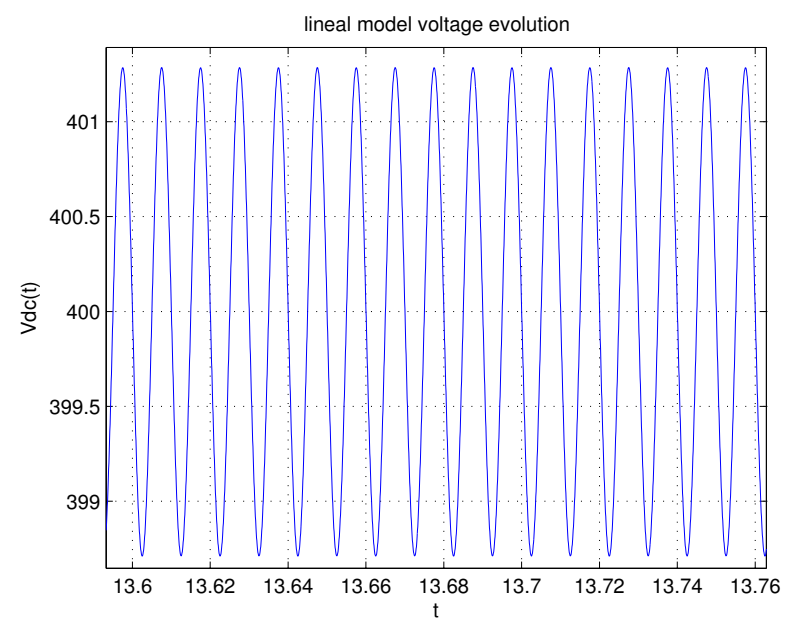

Fig. 8. Evolution of $V_{D C}$ in steady state.

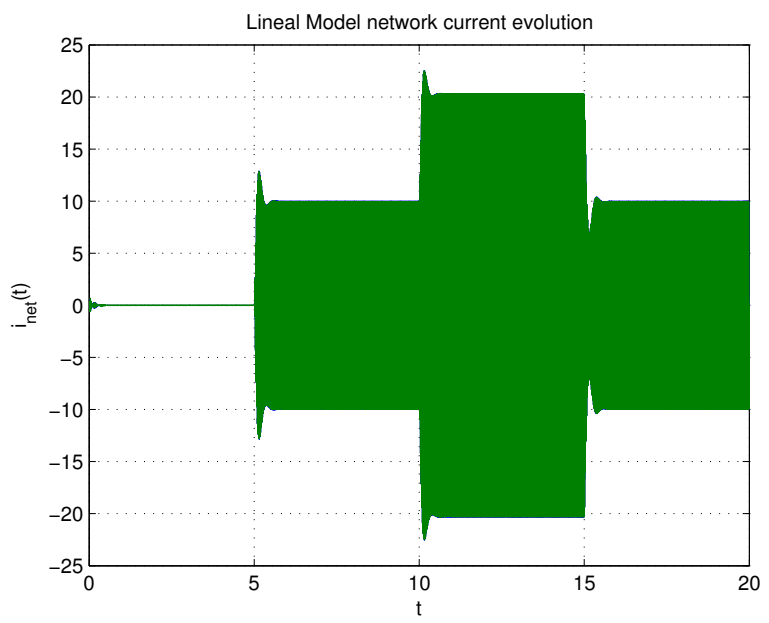

Fig. 9. Evolution of $x_{1}$ during the simulation.

\section{CONCLUSIONS}

In this paper, a resonator based control scheme for a single phase AC/DC rectifier system with unity power factor has been proposed. The paper suggests a cascade controller architecture: an inner control in charge on shaping the net current, based on a passive resonator architecture (the introduction of a resonator bank inside the control loop assures perfect asymptotic tracking so power factor 1 in steady state) and an outer control in charge of assuring the DC bus mean value. The proposed architecture has been validated by numerical simulations.

Differently from other control schemes, proposed scheme allows us to adapt the system frequency to the network frequency just by changing the value of $\omega$ in the resonator bank. This can be applied due to the passivity property of the current control system.

Currently, an experimental setup is being built in order to practically validate these theoretical and simulated results.

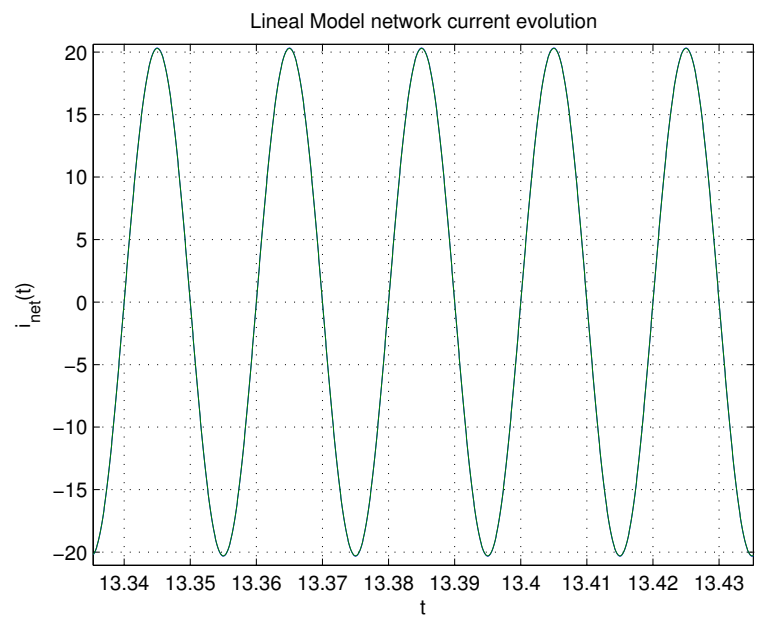

Fig. 10. Evolution of $x_{1}$ in steady state (both the current and the current reference).

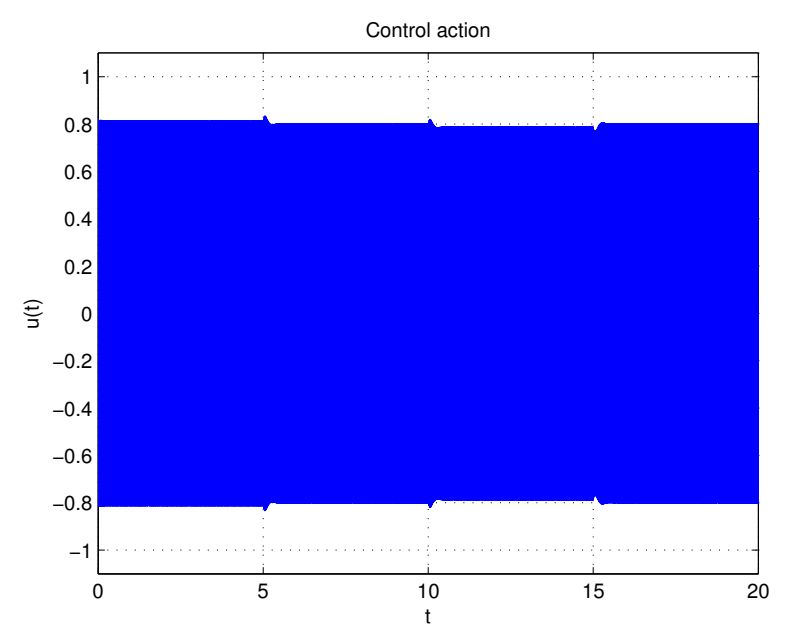

Fig. 11. Evolution of $u$ during the simulation.

\section{REFERENCES}

[1] R. Boys and A. W. Green. Current-forced single-phase reversible rectifier. IEE Proc., pt. B, 136, September 1989.

[2] G. Escobar, D. Chevreau, R. Ortega, and E. Mendes. An adaptive passivity-based controller for a unity power factor rectifier. IEEE Trans. on Control Systems Technology, 9(4):637-644, July 2001.

[3] B.A. Francis and W.M. Wonham. Internal model principle in control theory. Automatica, 12:457-465, 1976.

[4] Ned Mohan, Tore M. Undeland, and William P. Robbins. Power Electronics. Converters, Applications, and Design. John Wiley \& Sons, Inc., New York, second edition, 1995.

[5] E. Wernekinck, A. Kawamura, and R. Hoft. A high frequency ac/dc converter with unity power factor and minumum harmonic distortion. IEEE Trans. on Power Electronics, 6:364-370, July 1991. 